\title{
Correction to: Potential role of uncertainty relation for thermodynamic quantities in the Planck's cavity radiation
}

Shoichi Nagata ${ }^{a}$

Muroran Institute of Technology, 27-1 Mizumoto-cho, Muroran, Hokkaido 050-8585, Japan

Published online 10 September 2021

(C) EDP Sciences, SIF and Springer-Verlag GmbH Germany, part of Springer Nature 2021

Correction to: Eur. Phys. J. D (2021)75:216

https://doi.org/10.1140/epjd/s10053-021-00232-2

\begin{tabular}{lll}
\hline & Error & Correction \\
\hline Eq. (1) & $U \frac{1}{2} \mu_{0} H^{2}=\frac{1}{2} \varepsilon_{0} E^{2}$, & $\frac{1}{2} \mu_{0} H^{2}=\frac{1}{2} \varepsilon_{0} E^{2}$, \\
Eq. (50) & $\cdots+\frac{2 \pi}{h}<\Delta Q_{\nu} \Delta P_{\nu}>_{T^{2}}$ & $\cdots+\frac{2 \pi}{h}<\Delta Q_{\nu} \Delta P_{\nu}>_{T}^{2}$ \\
\hline
\end{tabular}

The original article can be found online at https://doi.org/ 10.1140/epjd/s10053-021-00232-2.

\footnotetext{
${ }^{\mathrm{a}}$ e-mail: nagatas@almond.ocn.ne.jp (corresponding author)
} 